\section{Konfrontation statt Elimination}

D en Paradigmenwechsel von der Allergenvermeidung hin zur Toleranzinduktion durch Konfrontation behandelt ein neues Buch von Imke Reese und Christiane Schäfer. Beide sind ausgewiesene Expertinnen im Bereich der allergologischen Diätetik. Das Buch stellt auch ein Stück Allergiegeschichte im Präventionsbereich dar - in fast allen Kapiteln wird eine Rückschau bzw. ein Vergleich zu den früheren Allergiepräventionsstrategien vorgenommen.

Kernstück sind aber die aktuellen Empfehlungen zur Prävention: Sie sind nach Kapiteln geordnet umfangreich beschrieben und jeweils mit dem entsprechenden Evidenzlevel versehen. Sehr ausführlich werden die einzelnen Themen inhaltlich in allen Facetten dargestellt und die Studienlage praxistauglich bewertet. Das Buch profitiert von der Mitarbeit einer der Autorinnen an der Neugestaltung 2009 der S3-Leitlinie zur Allergieprävention.
Hervorzuheben sind die Kapitel Ernährung, bei der ein revolutionärer Wandel stattgefunden hat: kein Verzicht mehr auf potenzielle, aber ernährungsphysiologisch wichtige Nahrungsmittelallergene - sondern gezielte Aufnahme in den Speiseplan der Schwangeren, Stillenden und der Säuglinge bei der Einführung der Beikost $a b$ dem vollendeten vierten Lebensmonat. Anschaulich und spannend ist auch der Stellenwert von Probiotika in der Allergieprävention dargestellt, wobei die $\mathrm{Da}$ tenlage kontrovers und somit die Diskussion darüber noch im Fluss ist.

Insgesamt gilt, dass der Leser auch in allen anderen Kapiteln wie Haustierhaltung, Innen- und Außenluftklima oder Hausstaubmilbensanierung verständlich informiert und dafür sensibilisiert wird, welche Maßnahmen sinnvoll sind.

Die Autorinnen haben die umfangreichen Daten, die zur Allergieprävention vorliegen, nicht nur sehr ausführlich dargestellt. Den beiden Autorinnen liegt auch die Praktikabilität im Alltag am Herzen: Davon zeugt das sehr persönlich geschriebene, vierte Kapitel. Das Buch endet visionär, in dem ein präventives Zeitfenster für Nahrungsmittelallergene analog zu den Studien bei der Zöliakie präsentiert oder die Möglichkeit einer Immunmodulation durch $\mathrm{Pa}$ rasiten in Aussicht gestellt wird.

Abschließend ist diesem gelungenen Buch zu bescheinigen, dass es für Ärzte und Therapeuten in der Allergologie, für Betroffene und für Eltern von Kindern mit und ohne Atopierisiko ein kompetenter und hilfreicher Ratgeber auf dem Weg der Allergieprävention ist. red

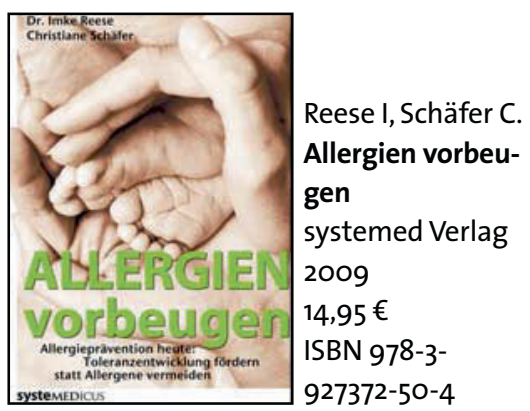

\section{Praktische Hilfe bei Laktoseintoleranz \& Co.}

U nerkannte Zuckerverwertungsstörungen verursachen oft quälende und unangenehme Beschwerden wie Blähungen, Durchfall oder laute Bauchgeräusche. In der Regel haben die Betroffenen einen langen Leidensweg hinter sich, bevor die Ursache der Symptome gefunden ist. Doch auch dann, wenn die Auslöser identifiziert sind, fehlt es häufig an praxistauglichen Tipps für die Umsetzung ernährungstherapeutischer Empfehlungen im Alltag. Vor allem in den Fällen, in denen mehrere Störungen parallel vorhanden sind - wenn beispielsweise neben einem Fruktose-Transportproblem auch noch die Spaltung der Laktose Probleme hervorruft - sind fundierte Ratgeber Mangelware.

Als Patient hatte man bislang nur die Möglichkeit, sich Rezepte und Tipps für ein erfolgreiches Krankheitsmanagement aus mehreren Ratgebern zusammenzubasteln. Das bedeutete im Zwei- felsfall eher Verwirrung als Hilfe. Denn nur wenige auf dem Markt erhältliche Ratgeber zu Zuckerverwertungsstörungen wurden von so versierten Fachkräften geschrieben, wie es bei dem eingespielten Autorenteam Christiane Schäfer und Anne Kamp der Fall ist. Jetzt gibt es den Ratgeber für Unverträglichkeiten gegenüber Fruktose, Laktose und Sorbit aus der Feder der beiden Autorinnen.

Das Buch enthält einen kurzen, aber sehr informativen und patientengerecht geschriebenen Überblick über die Erkrankungen, deren Ursachen, die richtige Diagnostik und eine praktische Hilfe für die Umsetzung ernährungstherapeutisch sinnvoller Maßnahmen. Die Autorinnen weisen ausdrücklich darauf hin, dass die Verträglichkeitsgrenze für die genannten Auslöser sehr unterschiedlich sein kann und deshalb individuell ausgetestet werden muss. Die Grundlage dafür bietet ein dreistufiger Diätplan. Hilfreich ist bei diesem Plan, dass auch solche Lebensmittel mit aufgenommen wurden, die Verdauungsprobleme verursachen können, obwohl sie keine der genannten Auslöser enthalten.

Den Hauptteil des Buches nehmen abwechslungsreiche und sehr schmackhafte Rezeptideen ein, die dem Patienten mit Hilfe attraktiver Fotos aufzeigen, dass eine leckere und genussvolle Ernährung auch mit einem eingeschränkten Speiseplan möglich ist. Die Rezepte sind nicht nur von Hobbyköchen umsetzbar, sondern auch für kochunerfahrene Patienten geeignet.

Dr. Imke Reese, München

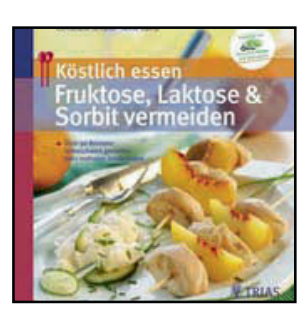

Schäfer C, Kamp A.

Fructose, Laktose

\& Sorbit

vermeiden

Trias Verlag 2009

$19,95 €$

ISBN 978-3-8304-

$3460-3$ 